\title{
Supersymmetry and the positron excess in cosmic rays
}

\author{
G.L. Kane ${ }^{a}$, Lian-Tao Wang ${ }^{a}$, James D. Wells ${ }^{b, c}$ \\ ${ }^{(a)}$ Physics Department, University of Michigan, Ann Arbor, MI 48109 \\ (b) Physics Department, University of California, Davis CA 95616 \\ ${ }^{(c)}$ Lawrence Berkeley National Laboratory, Berkeley, CA 94720
}

\begin{abstract}
Recently the HEAT balloon experiment has confirmed an excess of high-energy positrons in cosmic rays. They could come from annihilation of dark matter in the galactic halo. We discuss expectations for the positron signal in cosmic rays from the lightest superpartner. The simplest interpretations are incompatible with the size and shape of the excess if the relic LSPs evolved from thermal equilibrium. Non-thermal histories can describe a sufficient positron rate. Reproducing the energy spectrum is more challenging, but perhaps possible. The resulting light superpartner spectrum is compatible with collider physics, the muon anomalous magnetic moment, Z-pole electroweak data, and other dark matter searches.
\end{abstract}

PACS: 12.60 Jv, 13.40 Em , hep-ph/0108138, MCTP-01-37, LBNL-48801

Recent experiments: Good solutions to the cosmological dark matter problem often involve hypothesizing a stable weakly interacting massive particle (WIMP). The particles populate galactic halos providing gravitational support to the unusual constant velocity profiles of many galaxies.

Direct experiments continue to look for WIMPs scattering off nuclear target detectors. Indirect experiments rely on annihilation of ambient WIMPs that produce an excess above background of photons, anti-protons, positrons or neutrinos in cosmic rays. Each of these experiments has its unique experimental challenges, and its unique astrophysical assumptions and uncertainties. For example, to be successful the direct searches need a significant local density of WIMPs, whereas discovery of a monochromatic photon line from WIMP annihilations generally requires a cusping distribution near the galactic center. The charged particle signals $\left(\bar{p}\right.$ and $\left.e^{+}\right)$require an accurate model describing their propagation and energy loss from their source at WIMP annihilations in the galactic halo to the detector on earth.

Our imperfect understanding of the dark matter distribution and other astrophysics uncertainties makes it impossible to predict which signal would be the first to demonstrate evidence for WIMP dark matter. For this reason, all the different experiments designed for this purpose are interesting and necessary parts of a comprehensive search strategy. Once WIMPs are found all the experiments provide information about their properties and help to determine the WIMP relic density.

Recently, the HEAT collaboration has found tantalizing evidence for unexpected structure in the $e^{+} /\left(e^{-}+e^{+}\right)$ energy spectrum [1] [3]. The first set of data from the 1994-1995 flights indicated a rise or bump in the positron fraction at energies above about $7 \mathrm{GeV}$. Using a different instrument, with different systematics, the HEAT collaboration found in the data of their 2000 flight a similar rise. The consistency between the data sets adds further confidence in the measured energy distribution of the positron fraction.

Attempts at a standard supersymmetry interpretation: One of the most compelling theories for WIMP dark matter is supersymmetry. R-parity conserving supersymmetry naturally provides a dark matter candidate in the lightest supersymmetric partner (LSP).

In some models, such as "minimal supergravity", the LSP is mostly bino (fermion superpartner to the hypercharge gauge boson). For relatively light superpartners (mass near the weak scale), one finds in large fractions of the parameter space of these models that a simple thermal history calculation will give an answer remarkably close to the $\Omega h^{2} \simeq 0.1$ needed for an acceptable cold dark matter candidate. LSP annihilations into positrons can then be searched for in cosmic rays 国 6]. However, the standard supersymmetry model does not explain the HEAT data, for two reasons.

First, the positron excess is most simply produced by LSP annihilations into $W$ bosons [6 \&], one of which subsequently decays into a positron. However, binos do not couple to $W$ 's and so this final state is suppressed compared to other final states. There is still the option of producing positrons from cascade decays of the other final states of bino annihilation. For example, annihilations into tau leptons can produce positrons from leptonic decays of $\tau^{+}$or from fragmentation of $\tau$ jets. However, the total annihilation rate for binos is small. Although this is correlated with a reasonable $\Omega h^{2}$, the annihilation rate is insufficient to produce a large flux of positrons to overcome expected backgrounds. Therefore, the positron fraction signal is not expected to be visible, unless we have underestimated important astrophysical parameters considerably. The HEAT data is likely not explained by bino LSP theories where the relic abundance of LSPs is accurately computed from a simple thermal 
history of the universe.

Higgsino and wino dark matter: What is needed to explain the HEAT signal is a large relic abundance, a large annihilation rate, and a rising distribution of positron fraction at energies above about $7 \mathrm{GeV}$. Higgsino and wino LSPs may do this. They couple at full strength to the $W$ boson and have a large annihilation rate. As long as they have mass above $m_{W}$, higgsinos and winos will annihilate predominantly into $W W$ final states and so can produce a large number of high-energy positrons from $W \rightarrow e^{+}+X$. This has been discussed recently in the context of traditional supersymmetry models with large higgsino fraction LSP [7, \& and anomaly mediation with wino LSP 10$]$.

Since the relic abundance correlates inversely with the strength of annihilation, there is still the worry that there will be too few of these LSPs in the galactic halo to annihilate with each other and produce a signal. However, that argument is based on a standard thermal history calculation which predicts $\Omega_{\mathrm{LSP}} \lesssim 10^{-3}$. Non-thermal sources and non-standard cosmologies have been found to produce a significant relic abundance independent of the thermal annihilation rate [9]1]. It is one of the important conclusions of this paper that the higgsino dark matter density probably must be understood outside the normal thermal evolution framework if the HEAT data is indicating LSP annihilations.

Details of the positron signal: In order for the reader to understand our results we will briefly describe the assumed dark matter density profile we use to produce expected positron fluxes from LSP annihilations. The dark matter halo is assumed to be spherically symmetric isothermal sphere whose density at a position $r$ from the galactic center is

$$
\rho(r)=\rho_{0} \frac{a^{2}+r_{0}^{2}}{a^{2}+r^{2}}
$$

where $\rho_{0}=0.3 \mathrm{GeV} / \mathrm{cm}^{3}$ is the local LSP density, $a=3.5$ $\mathrm{kpc}$ is the core radius, and $r_{0}=8.5 \mathrm{kpc}$ is the distance of the earth from the galactic center.

The flux $F_{e^{+}}$of positrons at the detector for higgsino or wino can be calculated from

$$
\frac{d F_{e^{+}}}{d E}=\frac{\rho_{0}^{2}}{m_{\chi}^{2}} \int d \epsilon G_{e^{+}}(E, \epsilon) \sum_{f}(\sigma v)_{f} A_{e^{+}}^{f}(\epsilon)
$$

where $(\sigma v)_{f}$ is the annihilation rate of $\chi \chi$ into the final state $f=W W$ or $f=Z Z, G_{e^{+}}(E, \epsilon)$ is the positron propagation Green's function, and $A_{e^{+}}^{f}(\epsilon)$ is the average positron energy distribution function for the final state $f$ at the source (pre-propagation). The $A_{e^{+}}^{f}$ functions are normalized such that $\int d \epsilon A_{e^{+}}^{f}(\epsilon)$ is the average number of positrons in decays of the final state $f$.

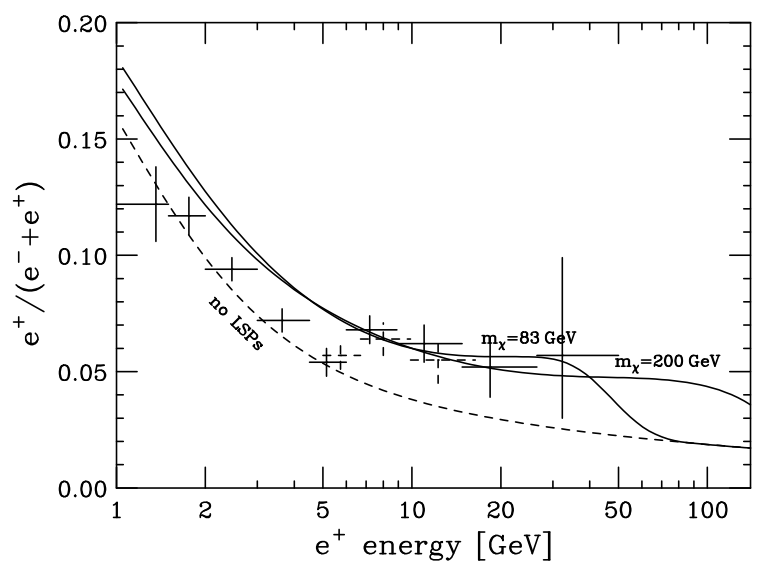

FIG. 1. Positron fraction as a function of energy. The lower dashed line is the expected signal fraction with no LSP annihilations for a certain set of astrophysical assumptions described in the text. The solid lines also include the positrons and electrons from the annihilations in the galactic halo of LSPs with mass $m_{\chi}=83 \mathrm{GeV}$ and $m_{\chi}=200 \mathrm{GeV}$, with boost factors of 2.7 and 3.9 respectively. The 1994-1995 HEAT data is represented by the solid line cross-hairs, and the 2000 HEAT data by the dashed line cross-hairs.

We utilize DARKSUSY [12] for calculating the flux. We also tested the results by simulating the $A_{e^{+}}^{f}(\epsilon)$ from Pythia. The Green's function $G_{e^{+}}(E, \epsilon)$ can be extracted from refs. [8,13]. The numerical values we used are from [8,12] with energy loss time $\tau_{E}=10^{16} \mathrm{sec}$, and with energy-dependent diffusion constant

$$
K(\epsilon)=6.1 \times 10^{27}\left(\frac{\epsilon}{1 \mathrm{GeV}}\right)^{0.6} \mathrm{~cm}^{2} \mathrm{sec}^{-1} .
$$

As indicated earlier, the thermal relic abundance is much too small to be of cosmological significance, but non-standard mechanisms can save the higgsino and wino as dark matter candidates. From here on we assume that the local density $\rho_{0}$ is made up entirely of neutralino dark matter, assuming a non-thermal source for the LSPs such as from late decays of very heavy gravitinos [9]. Then we no longer need to concern ourselves with neutralino relic abundance, since the value of $\rho_{0}$ captures all the information we need about LSP abundance in our positron flux calculation. An obvious consequence of this approach is that we do not rescale $\rho_{0}$ according to the thermal relic abundance calculation.

Fig. 1 1 shows the positron fraction energy distribution for the HEAT data [2,3], expected distribution with no LSP annihilations, and expected distribution with LSP annihilations subject to the above assumptions. The plot is made for higgsino/gaugino mixed scenario with LSP masses of $m_{\chi}=83 \mathrm{GeV}$ and $200 \mathrm{GeV}$.

We have normalized the positron distribution to 0.06 at $10 \mathrm{GeV}$ for each value of the LSP mass. To do this we had to arbitrarily multiply the flux calculated with 


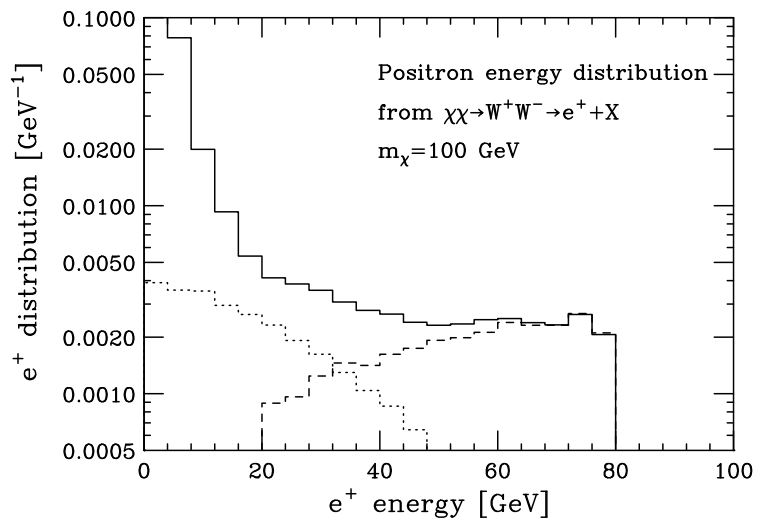

FIG. 2. The solid line is the average positron energy distribution from cascade decays resulting from LSP annihilations into $W$-boson pairs. The mass of the LSP is $100 \mathrm{GeV}$ in this example. The dashed line tracks the positrons from $W^{+} \rightarrow e^{+}$ direct decays, and the dotted line from $W^{+} \rightarrow \mu^{+} / \tau^{+} \rightarrow e^{+}$ direct decays.

the above assumptions by a factor ("astrophysical boost factor") of 2.7 and 3.9 for $m_{\chi}=83 \mathrm{GeV}$ and $200 \mathrm{GeV}$, respectively. An extra boost factor less than about 10 is probably well within the astrophysical uncertainties of parameters used to calculate the flux. This gives us confidence that low-mass LSPs $m_{W}<m_{\chi} \lesssim 200 \mathrm{GeV}$ are worth pursuing as possible interpretations of the positron energy distribution and the total flux.

The HEAT data appears to show a dip in the positron energy fraction near $E_{e^{+}}=7 \mathrm{GeV}$. A dip would indicate that a signal should have a large bump in its positron distribution at energy above $7 \mathrm{GeV} . \quad \chi \chi \rightarrow W^{+} W^{-}$ annihilations are the best hope to produce a bump in the positron spectrum from LSP annihilations, since $W^{+} \rightarrow$ $e^{+} \nu$ decays lead to a peak in the positron spectrum at high energies. However, there are numerous other sources for positrons in $W$ decays, including cascades from $\tau$ and $\mu$ leptons, and decays of pions in jets. In Fig. 2 we show the average positron energy distribution from $\chi \chi \rightarrow W^{+} W^{-}$annihilations, simulated using Pythia results from $e^{+} e^{-} \rightarrow W^{+} W^{-}$. The lack of a peak in this distribution clearly indicates that simple LSP annihilations cannot reproduce a strong peak in the positron energy spectrum.

Therefore, in order for the LSP annihilations to be consistent with the data we have to assume that there is no significant dip in the data, but rather a change in slope. Given the error bars for the HEAT data points, this possibility is not out of the question. In this case, the signal arises from an LSP-induced positron distribution that is somewhat flatter than the background positrons. The prediction is best fit to the data when the number of signal positrons starts to become a significant fraction of the total positron rate at $E_{e^{+}} \gtrsim 5 \mathrm{GeV}$.

We briefly mention here another interpretation of the data which is somewhat fine-tuned, but would be more consistent with strong peaking in the positron energy spectrum. The electron sneutrino is stable or nearly stable if its mass is extremely close to the LSP's. In this case one could imagine $\chi \tilde{\nu}^{*} \rightarrow W^{-} e^{+}$annihilations with the positron energy peaked at $E_{e^{+}}=m_{\chi}\left(1-m_{W}^{2} / 4 m_{\chi}^{2}\right)$. Numerically, to get a sharp peak at about $8 \mathrm{GeV}$ requires $m_{\tilde{\nu}}+m_{\chi} \approx m_{W}+10 \mathrm{GeV}$. Whether the neutralino or sneutrino is the lightest would not be important. We have not carefully studied this possibility, although we recognize that the LEPII collider data would severely constrain it, and maybe even rule it out.

Correlated phenomena: In the previous sections we have concluded that

- Traditional supersymmetry with thermal relic abundance near $\Omega h^{2} \simeq 0.1$ cannot yield an excess of positrons above background because the annihilation rate is too low,

- A higgsino or wino LSP with mass $m_{W}<$ $m_{\chi} \lesssim 200 \mathrm{GeV}$ could yield a significant excess of positrons above background provided the relic abundance is from a non-thermal source,

- A generic higgsino or wino interpretation is consistent with the HEAT data only if no strong dip is present,

- Uncertainties in the data and in astrophysical processes such as positron production, propagation and modulation means we may not need a newphysics interpretation of the HEAT data, although our current understanding suggests we do.

If the higgsino or wino interpretation of HEAT data is correct, we should expect other correlating phenomena that can be measured and quantified. First, it is wellknown by now that the recent excess 14 in the muon anomalous magnetic moment is consistent with light supersymmetry 15 17. Particularly, a light higgsino and large $\tan \beta$ are helpful to get a large supersymmetric correction, since the higgsino-smuon-muon vertex is a $\tan \beta$ enhanced chirality flip. The supersymmetric contribution to $\delta a_{\mu}$ with higgsino or wino LSP is

$$
\delta a_{\mu} \lesssim 14 \tan \beta\left(\frac{100 \mathrm{GeV}}{m_{\chi}}\right)^{2} \times 10^{-10}
$$

Equality in eq. (4) is attained when all other sparticle masses are very close to the LSP mass. The measurement minus the Standard Model contribution is $\delta a_{\mu}=(41 \pm$ 16) $\times 10^{-10}$. Therefore, moderate $\tan \beta$ and low mass superpartners have no difficulty recovering the central value for the measured $\delta a_{\mu}$.

Colliders can also search for higgsino and wino cold dark matter. These searches are notoriously difficult 
because there is no guarantee that visible superpartners have mass close to the LSP mass and are therefore accessible by the colliders. In the case of higgsinos and winos, there are charged particles nearby, $\tilde{H}^{+}$or $\tilde{W}^{+}$; however, they are almost degenerate in mass to the LSP. The production of $e^{+} e^{-} \rightarrow \tilde{H}^{+} \tilde{H}^{-}$may be high, but the final state of two soft pions from $\tilde{H}^{ \pm} \rightarrow \tilde{H}^{0} \pi^{ \pm}$is very difficult to find. Searches have been conducted, and the mass limits for these sparticles are about $m_{W}$ [18], just below the interesting region for the HEAT signal. Future lepton colliders will have a much higher mass reach, and hadron colliders will be useful if other superpartners are produced [19].

Other astrophysics experiments may also see evidence for wino or higgsino cold dark matter. Nuclear target detectors have limits that are already sensitive to wino or higgsino LSPs in some parts of parameter space [20]. However, these limits depend on squark masses, heavy Higgs boson masses, etc. which feed into the spinindependent nucleon-LSP scattering cross-section, and which have little to do with the positron fraction prediction. Therefore, it is difficult to predict how sensitive next generation cryogenic detectors will be to light higgsinos and winos. A possible signal not in conflict with our results has been reported at DAMA [21,22].

On the other hand, loop-induced annihilations of $\chi \chi \rightarrow \gamma \gamma$ are very high for higgsinos and winos. One therefore expects a monochromatic photon signal to arise from annihilations of winos and higgsinos in the galactic halo 23,24. As mentioned at the beginning, the astrophysical uncertainties of this calculation are quite different than the positron fraction calculation. Therefore, it is difficult to predict if experiments such as GLAST will see a signal, but we do expect so if the HEAT results are due to LSP annihilations.

Finally, it has been suggested recently that the precision electroweak data is more consistent with light superpartners 25]. Sneutrino masses below $m_{W}$ and slepton masses just above the experimentally allowed region are the most important requirements for the successful fit to data. Light gauginos are also helpful, but not as critical. Therefore our interpretation of the HEAT data, which requires superpartner masses near $m_{W}$, is not only compatible with the precision electroweak data, but may be encouraged by it. This is another reason why the LSP interpretation is worthwhile pursuing even though it has difficulty reproducing the precise structure of the data.

Acknowledgments: This work was supported in part by National Science Foundation, the Department of Energy, and the Alfred P. Sloan Foundation. We appreciate stimulating discussions with G. Tarle, S. Mrenna, and D. Chung, and help from T. Wang.
[1] S. W. Barwick et al. [HEAT Collaboration], Astrophys. J. 482, L191 (1997) astro-ph/9703192.

[2] S. Coutu et al., Astropart. Phys. 11, 429 (1999) astro$\mathrm{ph} / 9902162$.

[3] S. Coutu et al., "Positron measurements with the HEATpbar instrument," Proceedings of ICRC 2001.

[4] A. J. Tylka, Phys. Rev. Lett. 63, 840 (1989) [Erratumibid. 63, 1658 (1989)].

[5] M. S. Turner and F. Wilczek, Phys. Rev. D 42, 1001 (1990).

[6] M. Kamionkowski and M. S. Turner, Phys. Rev. D 43, 1774 (1991).

[7] J. L. Feng, K. T. Matchev and F. Wilczek, Phys. Rev. D 63, 045024 (2001) astro-ph/0008115.

[8] E. A. Baltz and J. Edsjo, Phys. Rev. D 59, 023511 (1999) astro-ph/9808243.

[9] T. Gherghetta, G. F. Giudice and J. D. Wells, Nucl. Phys. B 559, 27 (1999) hep-ph/9904378.

[10] T. Moroi and L. Randall, Nucl. Phys. B 570, 455 (2000) hep-ph/9906527.

[11] R. Jeannerot, X. Zhang and R. Brandenberger, JHEP 9912, 003 (1999) hep-ph/9901357.

[12] P. Gondolo, J. Edsjo, L. Bergstrom, P. Ullio and E. A. Baltz, astro-ph/0012234.

[13] I. V. Moskalenko and A. W. Strong, Phys. Rev. D 60, 063003 (1999) astro-ph/9905283.

[14] H. N. Brown et al. [Muon g-2 Collaboration], Phys. Rev. Lett. 86, 2227 (2001) hep-ex/0102017.

[15] L. L. Everett, G. L. Kane, S. Rigolin and L. Wang, Phys. Rev. Lett. 86, 3484 (2001) hep-ph/0102145.

[16] J. L. Feng and K. T. Matchev, Phys. Rev. Lett. 86, 3480 (2001) hep-ph/0102146.

[17] S. P. Martin and J. D. Wells, Phys. Rev. D 64, 035003 (2001) hep-ph/0103067.

[18] P. Abreu et al. [DELPHI Collaboration], Eur. Phys. J. C 11, 1 (1999) hep-ex/9903071.

[19] J. F. Gunion and S. Mrenna, hep-ph/0103167.

[20] B. Murakami and J. D. Wells, hep-ph/0011082.

[21] R. Bernabei et al. [DAMA Collaboration], Phys. Lett. B 480, 23 (2000).

[22] P. Belli et al. , Phys. Rev. D 61, 023512 (2000) hep$\mathrm{ph} / 9903501$.

[23] S. Mrenna, J.D. Wells, Pheno 2000 Plenary Talk, http://pheno.physics.wisc.edu/pheno00/wells.ps

[24] P. Ullio, JHEP 0106, 053 (2001) hep-ph/0105052.

[25] G. Altarelli, F. Caravaglios, G. F. Giudice, P. Gambino and G. Ridolfi, JHEP 0106, 018 (2001) [hepph/0106029; G. Cho and K. Hagiwara, Phys. Lett. B 514, 123 (2001) hep-ph/0105037. 